\title{
Audit of thromboprophylaxis in a district general hospital
}

\author{
Authors: Pratibha Machenahalli, ${ }^{\mathrm{A}}$ Srividya Narayanan, ${ }^{\mathrm{B}}$ Sriranganatha Akavarapu, ${ }^{\mathrm{C}}$ Raj Tandy and SM Abidi
}

\section{Aims}

To assess the inpatient thromboprophylaxis practice over a 1-month period.

\section{Methods}

Inpatient audit proforma from King's College haematology department was used. We assessed the thromboprophylaxis practice of the inpatient medical, surgical and maternity unit. A snapshot, once-only visit to different wards was conducted. We used the following criteria for assessing the risks of venous thromboembolism (VTE):

$>$ reasons for admission

$>$ timely assessment of risk of VTE

$>$ weight

> categorisation of the patient's VTE risk and risk of bleeding

$>$ type of thromboprophylaxis (dose)

$>$ use of anti-embolic stockings (AES)

$>$ practice of consenting

$>$ appropriateness of thromboprophylaxis.

\section{Results}

> A total of 273 patients' drug charts were reviewed over the 1-month period.

> Documentation of the weight was done appropriately in 187 patients. Of these, 23 patients weighed $<50 \mathrm{~kg}$, 157 patients weighed between $50-100 \mathrm{~kg}$ and 7 patients weight was $>100 \mathrm{~kg}$.

> Timeline of completion of VTE assessment:

a) within 12 hours of admission: 171

b) >12 hours of admission: 5

c) unsure of the time: 50

d) not done: 45.

> Categorisation of the patients as per the protocol at admission:

a) high risk of VTE with low risk of bleeding: 181

b) high risk of VTE with high risk of bleeding: 34

c) low risk of VTE: 27

d) not completed: 31 .
Of these, the medical patients had the following assessments: 28 (a), 137 (b), 25 (c), 3 (d).

> Surgical patients: AES were used in 17 patients; five patients were at high risk of bleeding and VTE-AES were not prescribed.

$>$ VTE risk assessment from physician's perspective at the time of audit: 210 patients were at high risk of VTE with low risk of bleeding, 22 patients were at high risk of VTE with high risk of bleeding, 38 patients were at low risk of VTE.

> Type of thromboprophylaxis:

a) Medications: dalteparin, 188; dalteparin and AES, 8; dalteparin and warfarin, 2; AES, 2; no thromboprophylaxis in 55 patients; warfarin alone in 15 patients; half the dose of dalteprin in renal impairment in one patient.

b) AES: 17 patients used AES. 253 patients didn't use AES: 188 patients had medical reasons (on alternative treatment protocol), not prescribed in 63 patients, not needed in five patients.

> Thromboprophylaxis was appropriately assessed in 193 patients and not in 77 patients.

> Consenting: only 22 patients were consented. In 195 patients there was no documentation of consenting. Consenting was not applicable in 36 patients.

\section{Conclusions}

This is a snapshot version of thromboprophylaxis practice in 2014. Recently, we have observed a lot more improvement in the VTE prophylaxis with the use of electronic patient prescription records. From the above data, it is clear that documentation needs to be improved and assessment of risk of VTE needs to be done more aggressively. AES is a very economical and not harmful method which can be used more widely to prevent the risk of VTE. Re-audit of the current practice of thromboprophylaxis is recommended.

\section{Conflict of interest statement}

None.

Authors: ${ }^{\mathrm{A} I m p e r i a l ~ C o l l e g e ~ L o n d o n, ~ U K ; ~}{ }^{\mathrm{B}}$ University Hospitals of Burton and Derby NHS Trust, UK; ${ }^{C}$ Northwest London Hospitals NHS Trust, UK 